Rapport - Société canadienne d'histoire de l'Église catholique

\title{
La survie de Mgr de Laval et sa cause de béatification
}

\section{Jean-Marie Fortier}

Volume 25, 1957-1958

URI : https://id.erudit.org/iderudit/1007444ar

DOI : https://doi.org/10.7202/1007444ar

Aller au sommaire du numéro

Éditeur(s)

La Société canadienne d'histoire de l'Église catholique

ISSN

0318-6148 (imprimé)

1927-7075 (numérique)

Découvrir la revue

Citer cet article

Fortier, J.-M. (1957). La survie de Mgr de Laval et sa cause de béatification. Rapport - Société canadienne d'histoire de l'Église catholique, 25, 79-90. https://doi.org/10.7202/1007444ar

Tous droits réservés $@$ La Société canadienne d'histoire de l'Église catholique, 1958
Ce document est protégé par la loi sur le droit d'auteur. L'utilisation des services d'Érudit (y compris la reproduction) est assujettie à sa politique d'utilisation que vous pouvez consulter en ligne.

https://apropos.erudit.org/fr/usagers/politique-dutilisation/ 


\section{La survie de Mgr de Laval et sa cause de béatification}

\section{Avertissement}

Quand nous ne mentionnons pas la page précise d'un texte cité, c'est que nous avons dû travailler sur des copies. Voici les explications des abréviations employées dans nos références:

ASS Paris : Archives Saint-Sulpice de Paris.

ASQ : Archives Séminaire de Québec.

AAQ : Archives Archevêché de Québec.

AN Paris : Archives Nationales de Paris.

AAS : Acta Apostolicæ Sedis.

Nous traiterons de cet objet d'étude en trois parties.

Nous verrons d'abord que François de Laval, à sa mort, a joui d'une réputation de sainteté formant halo autour de sa dépouille mortelle.

Ce renom ne disparaîtra jamais tout à fait. Si, au cours du dixhuitième siècle, il perd de son éclat, si le procès de béatification n'est pas alors instruit, il ne faut en rendre responsable ni le désintéressement, ni l'oubli. Les malheurs de la succession épiscopale à Québec sous le régime français, les graves événements politico-religieux qui se déroulent au Canada à la fin du XVIII ${ }^{\bullet}$ siècle, le peu de souci qu'ont en général les Canadiens de conserver par écrits le souvenir des gestes passés expliquent ce phénomène. Telle sera la teneur de notre deuxième partie.

Quand la stabilité de l'Eglise romaine sera garantie chez nous, quand nos communautés se seront relevées de leurs ruines, quand les études historiques auront jeté sur les grandes figures de notre passé une lumière plus vive, même si celle-ci est parfois un peu crue, la vénération que discrètement on leur portait, s'affichera bien haut et rêvera de ceindre leur front de l'auréole des bienheureux. Aucune des causes de nos fondateurs n'échappera à cette loi. L'une ou l'autre pourra cheminer plus rapidement; elles partiront toutes de dates voisines: 1877, pour celle de Marie de l'Incarnation (205 ans après la mort de la servante de Dieu) ; 1878, pour celle de la Bienheureuse Marguerite Bourgeois (178 ans après); 1830, pour Mère d'Youville (129 ans après); 1890, pour celle de $\mathrm{M}^{\mathrm{gr}}$ de Laval (172 ans après). Ce sera notre but, dans la troisième partie, de décrire le mouvement de cette cause autour de la date - pivot de 1890.

Le 6 mai 1708, dans une chambre de son séminaire, mourait François de Laval, premier évêque de Québec. Il comptait quatrevingt-cinq ans d'âge et soixante et une années de sacerdoce. Préconisé, 
en 1658, vicaire apostolique de la Nouvelle-France sous le titre d'évêque de Pétrée, il devenait évêque de Québec, le $1^{\text {er }}$ octobre 1674. Démissionnaire pour raisons de santé, Monseigneur l'Ancien - c'est le titre qu'on lui donnera désormais - passera dans une quasi-retraite les vingt dernières années de sa vie.

Il était la personnalité ecclésiastique marquante du pays surtout depuis que les hasards de la guerre tenaient $\mathrm{M}^{\mathrm{gr}}$ de Saint-Vallier prisonnier en Angleterre. On pouvait bien s'attendre à ce que le décès du prélat causât quelque émotion dans la société canadienne du temps. Se contenterait-on de louer les mérites incontestables d'un pontificat long de cinquante ans? Les oraisons funèbres (ce genre littéraire d'une interprétation si délicate pour l'historien), prononcées à Québec et à Montréal, ici par Monsieur de la Colombière et là par Monsieur de Belmont, seraient-elles seules à publier les vertus, à célébrer les travaux de l'évêque défunt?

Une première série de témoignages sont les lettres de condoléances que le gouverneur de la Nouvelle-France, les gouverneurs de Montréal et de Trois-Rivières, les supérieurs des principales communautés religieuses du pays adressèrent aux autorités du Séminaire de Québec. Nul ne s'étonnera de trouver dans ces lettres de circonstance l'éloge du défunt: les écrits de cette nature se font une loi de consoler leurs destinataires en exhaltant les vertus de la personne pleurée. Nos correspondants sont trop polis pour s'affranchir de ce devoir. Ils auraient pu cependant respecter toutes les convenances sans mettre l'accent, avec une telle insistance, sur la sainteté du disparu et sans relater les faveurs obtenues grâce à son intercession. Qu'il me soit permis de lire quelques extraits de l'une ou l'autre lettre. Monsieur de Belmont, supérieur du Séminaire de Saint-Sulpice à Montréal écrit à Monsieur de Maizerets, du Séminaire de Québec:

- Vous ne pouvez pas douter que tout le Séminaire et moi n'ayons pris toute la part que nous devons à la perte commune que vous et nous avons faite d'un très saint et très bon père et d'un très illustre prélat. J'annonçai en chaire le jour de l'Ascension l'article de votre lettre où vous me donniez nouvelle de cette précieuse mort... On m'a dit, et le bruit en court ici, que M. Sarrazin, guéri par le baiser des pieds de Monseigneur, a paru par les rues, lui de qui on attendait la mort. Le saint prélat est sans doute bien digne de ce miracle; mais je me réjouis doublement de celui-là et l'on peut dire qu'en ressuscitant $M$. Sarrazin il ressuscite cent morts, à la fois, c'est-à-dire tous ceux à qui il rendra la santé 1 .,

Une première lettre de la supérieure de l'Hôtel-Dieu de Montréal s'était égarée; la Sœur Gaillard en rédige une deuxième:

- Je n'avais garde de manquer à vous témoigner et à tous vos Messieurs la particulière part que nous avons prise à votre juste douleur, perdant ici-bas un saint, un père et un évêque qui donnait à votre Séminaire un beau lustre par la sainteté de sa vie. Notre communauté, avait pour Sa Grandeur une vénération toute particulière, quoique la

1 ASS Paris, Documents pour servir à l'histoire de l'Eglise du Canada, vol. I, ff. 23-25. 
plus grande partie de mes sœurs n'aient jamais eu l'honneur de le voir. Mais nous avons toujours conservé dans notre communauté le respect et l'estime que nos mères nous ont inspirés de la sainteté et de la bonté de ce digne prélat... Nous avons reçu avec bien du respect un morceau de ses habits et un peu de ses cheveux. Le même soir que nous les reçumes, nous avons un malade qui ne repose ni jour, ni nuit, qui me demande de ces reliques. Je les lui donnai et il dormit toute la nuit et s'est toujours porté de mieux en mieux. Nous ne doutons pas que ce saint prélat ne vous soit et à nous toutes un grand et puissant protecteur et avocat auprès de Notre-Seigneur. Demandez pour nous, Monsieur, que nous puissions imiter ses vertus..., mais, sur toutes, sa profonde humilité et sa vie cachée ${ }^{2}$."

"C'est un saint en paradis qui intercédera pour nous ${ }^{3}$." «... le regardant comme un saint protecteur que nous avons dans le ciel, où il n'oubliera jamais le pauvre Canada ${ }^{4}$...» Telles sont les expressions de Monsieur de Vaudreuil et de Monsieur de Ramesay, respectivement gouverneur général de la Nouvelle-France et gouverneur de Montréal.

Si le critique ne parvient pas à laver cette correspondance sincère peut-être, mais officielle, de tout soupçon d'une complaisance émue, qu'il regarde agir le menu peuple. Celui-ci n'a aucun intérêt à ménager. Son sens chrétien le pousse à vénérer la sainteté authentique. Ce malade de l'Hôtel-Dieu de Montréal n'avait peut-être jamais vu $\mathrm{M}^{\mathrm{gr}}$ de Laval, à peine apprend-il que les religieuses ont reçu quelques cheveux et un morceau des habits du défunt, qu'il les réclame comme des reliques dont l'attouchement le guérira, espère-t-il. A Québec, le mouvement de piété populaire fut très vif. Trois témoins, indépendants l'un de l'autre, nous ont décrit ce va-et-vient de la foule auprès de la dépouille exposée en chapelle ardente. Une Ursuline de Québec écrit à la supérieure du monastère de Paris:

« Une barque qui part d'ici pour Plaisance me donne de vous faire savoir, que Notre-Seigneur a retiré à lui Monseigneur notre ancien évêque le sixième de mai... Sa mort ayant été aussi précieuse que sa vie a été sainte, laisse toute la Nouvelle-France embaumée de l'odeur de ses vertus... Le Ciel l'ayant avantagé de tout ce qui peut faire un grand saint, continue après sa mort de manifester la grandeur de ses vertus par le prompt soulagement et guérison que plusieurs personnes ont ressentis se recommandant à lui, et par un renouvellement intérieur que plusieurs ont ressenti à sa mort et qui continue encore. L'on ne peut attribuer qu'à un mouvement surnaturel l'extrême empressement que les peuples ont eu à lui faire toucher leurs chapelets, et surtout les petits enfants qui l'appelaient hautement le saint, s'approchant de lui sans crainte ni frayeur. Aussi n'a-t-il point changé après sa mort, son corps étant demeuré aussi souple et maniable, l'espace de huit jours, qu'il était durant sa vie... La cérémonie étant faite (les funérailles à la cathédrale), l'on ne déposa point le corps à l'endroit où on devait le mettre, le cercueil de plomb n'étant pas achevé ni l'endroit pour le placer disposé comme on le souhaitait. Messieurs du Séminaire le gardèrent encore cinq jours sans aucune mauvaise odeur, ni changement dans son visage, ni dans la souplesse de son corps. Ils tirèrent son visage en plâtre et, ce qui se trouve admirable, c'est qu'ayant retiré le moule, son visage devint d'une si grande beauté avec un rouge qui lui était naturel 5 .»

2 ASS Paris, Ibid.

3 ASS Paris, Ibid.

4 ASS Paris, Ibid.

5 ASS Paris, Ibid. 
Le témoin le mieux placé fut sans conteste le frère Hubert Houssart. Serviteur personnel de $\mathbf{M}^{\mathrm{gr}}$ de Laval depuis vingt ans, il n'avait pas attendu le décès de son maître pour ramasser tout ce qu'il pouvait de ce qui avait appartenu à l'évêque. Le jour même de la mort, un chirurgien pratiqua sur le cadavre une opération fréquente à l'époque: l'ablation du cour qui serait déposé dans une cassette de plomb. Le frère Houssart fait tremper des linges dans le sang de la plaie, détache quelques fragments d'os de dessus sa poitrine, coupe des cheveux et conserve ses habits et tout cela pour servir de très précieuses reliques ${ }^{6}$.

* Je crois, Monsieur, écrit-il à M. Tremblay, procureur du Séminaire de Québec, à Paris, je crois que vous et toutes les personnes bien intentionnées approuverez mon procédé en cela, comme effectivement plus de trois mille personnes de toute sorte d'état et de condition l'ont déjà approuvé en Canada, en demandiant avec empressement et s'estimant heureuses d'avoir de petites parcelles du dit linge et de ces précieux restes de mon dit Seigneur, qu'ils portent sur eux avec respect et dévotion; des capitaines même et officiers de troupe ont fait faire exprès des reliquaires d'argent pour y en enfermer et les porter sur eux, étant mus à cela par l'idée et l'estime générales que chacun a du grand mérite et de la haute sainteté de mon dit Seigneur et par les secours extraordinaires et miraculeux que plusieurs ont reçus et reçoivent journellement dans leurs infirmités par l'invocation de mon dit Seigneur en s'appliquant des dites reliques ou les portant sur eux ${ }^{7}$.,

Enfin l'intendant Jacques Raudot relata les événements à son supérieur, le ministre de Seigneley:

- Depuis le départ des vaisseaux, il n'est rien arrivé ici qui mérite de vous être mandé que la mort de M. de Laval, ancien évêque de cette ville. Aussitôt après sa mort, les peuples l'ont pour ainsi dire canonisé, ayant eu la même vénération pour son corps, qu'on a pour ceux des saints, étant venus en foule de tout côté pendant qu'il a été exposé, et sur son lit de parade et dans l'église, lui faire toucher leurs chapelets et leurs heures. Ils ont même coupé des morceaux de sa robe que plusieurs ont fait mettre dans de l'argent et ils les regardent comme des reliques ${ }^{8.0}$

Nous n'avons pas ici le cas d'une compassion émue soucieuse de panser une plaie trop vive, ni une amitié intéressée, désireuse d'être payée de retour. Il s'agit d'un rapport transmis par un fonctionnaire subalterne à son chef de service, qui n'était pas susceptible de s'intéresser plus que cela au renom de sainteté de l'ancien évêque de Québec.

\section{II}

Cette vénération posthume fut-elle flambée éteinte aussitôt qu'allumée? Il semble que nous pouvons répondre non. Jusqu'à la fin du $\mathrm{XVIII}^{\circ}$ siècle, nous en découvrons des traces.

D'abord Monsieur Tremblay, procureur du Séminaire de Québec à Paris, ne reste pas inactif. Il a confié au graveur Duflos la reproduction d'un portrait de $\mathrm{M}^{\mathrm{gr}}$ de Laval peint en France en 1688. Il travaille

- ASQ, Lettres P, n. 102, p. 2.

7 ASQ, Lettres P, n. 102 p. 11.

8 AN Paris, Colonies (11G, vol. 3, ff. 181-182). 
à la rédaction d'un éloge de l'évêque disparu et réclame du Séminaire de Québec une documentation. Le 22 mai 1710, il écrit à Monsieur de Maizerets:

- Je n'ai pu recevoir de vous, l'an passé, aucune remarque ni réflexion sur ce que je vous mandais qu'on désirait de savoir pour achever de dresser l'éloge de notre cher ancien évêque de Québec. Je vous envoie cette année par deux voies copie de ce que l'on avait commencé à dresser de cet éloge. Vous aurez la bonté de nous envoyer quelques mémoires sur ce que vous croyez qu'on y doit ajouter ou diminuer... On prendra sur ce que vous enverrez et sur ce que nous avons déjà de quoi achever cet éloge que je crois que nous devons toujours faire imprimer, quand ce serait un peu tard, pour conserver le souvenir de ce saint prélat. Je vous ai envoyé de ses images l'an passé, qui restèrent à $\mathrm{La}$ Rochelle (ce) dont je fus fort mortifié. J'en ai donné encore cette année à nos Messieurs. Je souhaite qu'ils (sic) arrivent à bon port et vous pourrez les répandre, car je vous en enverrai à mesure que vous en aurez besoin $\mathbf{9}_{\text {." }}$

En 1711, les images ne partent pas; la navigation n'est pas sûre.

- Je ne vous envoie pas pour cette année d'images de notre cher ancien prélat. Le Frère Hubert Houssart prétend que nous avons mal fait de ne pas nous servir du tableau de Madame de Champigny qu'on a fait très grossièrement et que ces images ne lui ressemblent pas. Le tableau sur lequel on les a gravés (sic) lui ressemblait bien en 1688. Je vous en enverrai une autre année où il y aura moins de risques. Tout de même j'attends la paix pour vous envoyer le reste des offices de la Sainte-Famille que j'ai toujours dans mon armoire ${ }^{10} . \gg$

La paix revenue par le Traité d'Utrecht, le chanoine Thiboult s'embarque pour la France afin de plaider la cause du chapitre de Québec contre des statuts que veut lui imposer $\mathrm{M}^{\mathrm{gr}}$ de Saint-Vallier. En 1715, il fait antichambre chez le ministre Pontchartrain et voit son affaire remise à des commissaires. Il gagne sa cause: les anciens statuts ne seront pas abolis. Un des arguments qui remportèrent la victoire, fut la sainteté de leur auteur, l'ancien évêque de Québec.

MM. les commissaires convinrent qu'il ne fallait plus penser à ce projet de statuts ou du moins qu'on ne pouvait rien faire qu'on eût vu ceux qui avaient été dressés par l'ordre de Monsieur de Laval... qu'il n'y fallait plus penser vu qu'on trouvait que les réflexions que j'avais fait (sic) dessus les détruisaient absolument. Voici l'abrégé des principales: qu'il ne fallait point de nouveaux statuts puisque nous en avions de Monsieur de Laval qui était un saint 11.,

Le Père de la Chasse, jésuite, eut la tâche délicate de prononcer en février 1728 l'oraison funèbre de $\mathrm{M}^{\mathrm{gr}}$ de Saint-Vallier enseveli dans les circonstances douloureuses que l'on sait. L'orateur n'eut pas de peine à trouver des traits qui illustreraient les trois vertus du défunt: sa piété, son zèle et sa charité. Il ne lui était guère facile de passer sous silence la figure du prédécesseur. Voici en quels termes il l'évoque: "Nommé coadjuteur de l'illustre François de Laval de Montmorency, premier évêque de Québec, qui n'aurait peut-être jamais eu son semblable en

9 ASQ, Lettres 0, n. 50, pp. 19-20.

10 ASQ, Lettres 0 , n. 51, p. 27.

11 AAQ, ch. VIII, 25. 
piété, en zèle, en charité entre les évêques du Canada, si Jean-Baptiste de la Croix de Saint-Vallier n'avait été son successeur,... (La piété, le zèle et la charité !) Voilà ce qui l'égalera aux Laval et aux noms les plus fameux en vertu qu'il plaira à Dieu de donner à cette Eglise ${ }^{12}$.» Concédons que l'orateur sacré ait, dans une mouvement d'éloquence, engagé un peu vite la postérité. Concédons même qu'il était souverainement habile de confronter les deux évêques. Il reste que $\mathrm{M}^{\mathrm{gr}}$ de Laval lui paraît très grand. Ses successeurs, dans la pensée de l'auteur, pourront l'imiter, voire même l'égaler; il leur sera difficile de le dépasser.

Arrive au pays, en $1729, \mathrm{M}^{\mathrm{gr}}$ Dosquet accompagné d'un jeune prêtre brillant et promis à une œuvre littéraire abondante, Bertrand de la Tour. Le passage de ce dernier à Québec sera bref. Il s'intéressa à la personnalité de $\mathrm{M}^{\mathrm{gr}}$ de Laval mort depuis vingt et un ans seulement. Il interviewa, dirions-nous, les témoins oculaires des dernières années de $\mathbf{M}^{\mathrm{gr}}$ l'Ancien: il en témoigne d'ailleurs lui-même explicitement: "C'est sur de bons mémoires et sur le rapport d'un grand nombre de personnes qui avaient connu le saint évêque, que l'auteur, durant son séjour à Québec, a rassemblé les faits divers dont il rend compte au public... J'ai vu cinq à six frères et autant de sœurs - il s'agit ici des donnés du Séminaire de Québec - qui m’ont parlé avec une vénération infinie de leur ancien maitre dont ils m'ont appris bien des particularités que j'ai insérées dans cette histoire ${ }^{13}$.»

En 1748, $\mathrm{M}^{\mathrm{gr}}$ de Pontbriand ordonne d'exhumer les corps de $\mathbf{M}^{\mathrm{gr}}$ de Laval et de $M^{\text {gr }}$ de Lauberivière pour les inhumer de nouveau dans le chœur de la cathédrale.

A la fin du XVIII ${ }^{e}$ siècle, nous rencontrons deux textes où la mémoire de Laval est rappelée avec éloge. Le 25 janvier 1786, un amant des antiquités canadiennes, probablement un prêtre du Séminaire de Québec, entreprend de transcrire un vieux registre où se trouvent consignés des faits concernant l'histoire du séminaire depuis 1663 à 1721. Il le transcrit fidèlement. Quand le copiste ajoute au texte des notes personnelles, il a soin de les en bien distinguer. Ce sont ces notes qui nous intéressent présentement. Le copiste commente ainsi la notice relative au second incendie du Séminaire en 1705: "Ce fâcheux accident dut être bien sensible en particulier pour $\mathrm{M}^{\mathrm{gr}}$ de Laval, qui en quatre ans vit deux fois détruire son ouvrage. Il vécut, depuis ce second incendie, trois ans. Il est à croire que cet espace de trois années lui suffit, pour voir son cher séminaire renaître encore une fois de ses cendres et y terminer sa sainte carrière le 6 may $1708^{14}$." En 1794, l'abbé Octave Plessis prononça l'oraison funèbre de $\mathrm{M}^{\mathrm{gr}}$ Briand. Le parallélisme entre la vie des Laval et des Briand était frappant: l'un avait été le premier évêque sous le régime français, l'autre le premier sous le régime anglais. Tous deux avaient consacré à la retraite les dernières années d'une longue vie. Ces traits de ressemblance n'échappèrent pas aux regards perspicaces de l'abbé Plessis.

12 AAQ, Ev. de Q. I, n. 24, $\mathrm{I}^{\text {er }}$ cahier, p. 21; $\mathrm{II}^{\mathrm{e}}$ cahier, p. 6.

13 Louis Bertrand de Latour, Mémoires sur la vie de M. de Laval, premier évêque de Québec, vol. I. Cologne, Mortiens, 1761, p. IV et p. 100.

14 ASQ, Mss 6, p. 26 
" Mes Frères, où trouverez-vous des exemples d'un zèle aussi prévoyant des mesures aussi sages pour perpétuer le royaume de JésusChrist en Canada? Remontez dans l'histoire de cette Eglise (l'orateur repasse alors tous les évêques de Québec)... vous ne trouverez à vous arrêter qu'au fondateur de cette Eglise, au premier de ses pontifes. Dans M. de Laval seul, vous rencontrerez ce courage infatigable, cette étendue de desseins, cette prévoyance habile, ce génie créateur que tout le monde a admiré dans M. Briand ${ }^{15}$."

En France paraissent des œuvres historiques à caractère tantôt général, tantôt particulier. Il n'est pas difficile d'y découvrir, à l'égard de $\mathrm{M}^{\text {gr }}$ de Laval, la renommée persistante d'une vie sainte, "Obiit autem Quebeci die sexta Maii anno millesimo septingentesimo octavo non sine fama sanctitatis " consigne la Gallia Christiana ${ }^{16}$. Le Père de Charlevoix se devait de louer le premier évêque de Québec dans son Histoire de la Nouvelle-France. Aussi n'invoquerons-nous pas cet ouvrage. Retenons plutôt un extrait de sa Vie de Mère Marie de l'Incarnation éditée en 1735. Ce passage a le ton d'une confidence émue née à l'évocation d'une personne aimée.

- Nous l'avons vu ce saint prélat, dans ses dernières années conservant encore cette simplicité évangélique, qui rendait si respectable les premiers successeurs des apôtres; et nous avons eu la consolation, en recueillant ses derniers soupirs, de voir terminer par une sainte mort, une vie toute consacrée aux plus pénibles travaux de l'apostolat 17."

Le grand dictionnaire historique de Moréri au moins dans son édition de 1746 contient sur le serviteur de Dieu une notice intéressante qui se termine ainsi: "il mourut à Québec âgé de 86 ans, universellement regretté de ses peuples qui étaient charmés de ses vertus; et il opéra même des miracles à son tombeau ${ }^{18}$.» L'abbé Ladvocat consigne dans son dictionnaire historique publié en 1777: "François de Laval s'y (à Québec) fit estimer de tout le monde par sa vertu et son éminente piété ${ }^{19}$.»

En 1761 avait paru à Cologne chez Jean-Frédéric Mortiens l'ouvrage amorcé trente ans plus tôt par le chanoine Bertrand de la Tour. Ce fut la première biographie de $\mathbf{M}^{\mathrm{gr}}$ de Laval. Elle s'intitulait: Mémoires sur la vie de $M$. de Laval, premier évêque de Québec. La préface du livre évoquait une réalité périmée: Québec, résidence du gouverneur français. Si les négociations diplomatiques n'avaient pas encore déterminé à quelle allégeance serait soumis le Canada, les armes avaient tranché le débat. La victoire était restée à l'armée anglaise. Nous touchons ici à l'une des raisons pour lesquelles la cause du serviteur de Dieu ne fut et ne put être introduite plus tôt en cour de Rome. Déjà en 1711, les relations anglo-françaises étaient mauvaises. Rappelons-nous que $M$. Tremblay n'envoie pas d'images, cette année-là, car les risques sont trop grands et les voies maritimes peu sûres. Il attend le retour de la paix

AAQ, D. Québec 6, n. 16 p. 16-17.

Gallia Christiana, vol. II, Lutetiæ Parisiorum 1720, vol. 170.

17 De Charlevoix, La vie de la Mère Marie de l'Incarnation, Paris, Le Mercier, 1735 , p. 368.

19 Louis Moreri, Le grand dictionnaire historique, vol. V, Paris, 1746, p. 441.

19 Ladvocat, Dictionnaire historique..., vol. II, Paris, Didot, 1777, p. 39 de la lettre L. 
pour expédier ces offices de la Sainte-Famille qu'il a dans son armoire. Le traité d'Utrecht (1713) inaugure une période de tranquillité relative. C'est la bonace avant l'orage. En 1744, commence la guerre entre la France et l'Angleterre, guerre qui, terminée par le traité de Paris (1763), sanctionna la perte du pays aux mains de l'Angleterre. Assurer la perpétuité d'un épiscopat romain dans une colonie soumise à une métropole protestante fut l'une des tâches primordiales des évêques canadiens jusque dans les premières décades du dix-neuvième siècle ${ }^{20}$. Après le collège des Jésuites, que la conquête fait disparaître, c'est le Séminaire de Québec qui, de toutes les institutions religieuses de la Nouvelle-France subit le plus profondément les conséquences de cette conquête ${ }^{21}$.

Sous le régime français, l'Eglise de Québec avait été éprouvée. A la mort de $\mathrm{M}^{\mathrm{gr}}$ de Laval, l'évêque en titre de Québec était prisonnier en Angleterre. Revenu en Canada en 1713, après une absence de 13 ans, $M^{\text {gr }}$ de Saint-Vallier mourra 14 ans plus tard. De ses deux successeurs immédiats, $\mathbf{M}^{\mathrm{gr}}$ Duplessis-Mornay ne vint jamais à Québec et $\mathbf{M}^{\mathrm{gr}}$ Dosquet y vécut à peine deux ans. Toutes les espérances s'étaient reportées sur un nouvel évêque âgé de vingt-huit ans: $\mathrm{M}^{\mathrm{gr}}$ de Lauberivière. La Providence le montra au pays plutôt qu'elle ne le lui donna: sept jours après son arrivée le jeune pontife mourait emporté par les fièvres. Le pontificat de $\mathrm{M}^{\mathrm{gr}}$ de Pontbriand fut long (1741-1760) et tumultueux. Il ne connut presque pas la paix et la guerre devait le chasser de sa ville épiscopale bombardée pour le mener à Montréal où il mourut incertain du sort des armes françaises.

Notre copiste anonyme cité plus haut fait précéder ses transcripta d'une préface. Celle-ci est un petit traité sans prétention de méthodologie historique. Il souligne avec force la souveraine importance des sources écrites pour l'histoire et déplore l'insouciance de ses contemporains à leur égard. Laissons-le parler:

- Avant d'ouvrir mon régistre ou mémoire, qu'on me permette de dire en passant, un mot sur notre indifférence pour ce qui concerne l'antiquité: indifférence dont nos pères nous ont faits héritiers, et que nous lèguerons probablement à ceux qui nous survivront. Comme nous touchons encore quasi à la formation de la province, que quelques-

20 Nous avons relevé les mandements d'entrée des évêques de Québec depuis celui de $\mathrm{M}^{\mathrm{gr}}$ Briand, du 10 mars 1774, jusqu'à celui de $\mathrm{M}^{\mathrm{gr}}$ Signay, datant du 25 février 1833. Tous mentionnent le choix immédiat d'un coadjuteur pour assurer au siège de Québec la continuité de la succession épiscopale. Aucun mandement d'entrée n'est donné de $\mathbf{M}^{\mathrm{gr}}$ Hubert et de $\mathbf{M}^{\mathrm{gr}}$ Denaut. Tous deux cependant se choisissent aussi un coadjuteur. Le mandement d'entrée de $\mathrm{M}^{\mathrm{gr}}$ Plessis, daté du $1^{\text {er }}$ février 1806, est très significatif de l'incertitude où l'on était même à cette époque, plus de quarante ans après le traité de Paris. L'évêque éprouve le besoin de tranquilliser l'âme inquiète de ses fidèles: « Vous avez craint que les circonstances du moment ne permissent pas de procéder à la nomination d'un coadjuteur. Vous avez frémi à la pensée diun délai qui pouvait vous exposer de rester sans évêque, dans le cas où il plairait à Dieu de nous retirer du monde. Calmez vos inquiétudes, s... (Mandements des Evêques de Québec, T. III, p. 14). $\mathbf{M}^{\mathrm{gr}}$ Plessis annonce que l'administrateur civil des pays lui a * accordé " pour coadjuteur, Messire Bernard-Claude Panet.

21 Marcel Trudel, L'Eglise canadienne sous le régime militaire, 1759-1764, T. II: Les Institutions, p. 96. 
uns même qui vivent encore parmi nous, ayant vu le premier évêque du Canada et de ses premiers généraux, ou ayant pu en entendre parler à ceux qui les avaient vu, on se persuade sans façon qu'il en sera toujours de même. C'était aussi le sentiment de nos pères. En conséquence, on a négligé, et on néglige tous les jours de tenir acte des faits dans les formes, ou si on en dresse, ou qu'on en trouve de dressés, quel cas en fait-on? On les laisse périr, on les brûle, on les donne... Nous y perdons cependant plus que nous croyons, et par malheur, les remèdes ne seront plus de saison quand on s'apercevra du mal ${ }^{22}$.s

Difficultés politico-religieuses de l'Eglise du Canada, succession épiscopale mouvementée sur le siège de Québec, peu d'attache aux gestes du passé, voilà quelques faits qui retardèrent la cause sans faire perdre le souvenir du pontife.

En 1880, le révérend Edmond Langevin, vicaire général du diocèse de Rimouski, comparaît comme témoin au procès ordinaire. Interrogé sur la réputation de sainteté de $\mathbf{M}^{\mathrm{gr}}$ de Laval, il atteste que, depuis sa jeunesse, il a entendu parler souvent du serviteur de Dieu. Toujours l'on prononça devant lui ce nom avec respect. Il avait eu successivement comme directeurs, au grand séminaire, les abbés Joseph Aubry et Léon Gingras. Ces deux prêtres lui avaient inculqué une profonde vénération envers le premier évêque de Québec. Le jeune abbé Langevin fut secrétaire à l'évêché pendant ses études théologiques, peut-être pendant toute leur durée: soit de 1842 à 1847. Les évêques qu'il y servit alors, Nosseigneurs Signay et Turgeon l'entretenaient des vertus de leur illustre prédécesseur ${ }^{23}$. L'abbé Louis-Edouard Bois témoigne à son tour qu'il avait environ quinze ans quand un prêtre de l'évêché de Québec lui parla de $\mathrm{M}^{\mathrm{gr}}$ de Laval. Puisque le témoin était né en 1813, nous avons ici une indication pour les années $1830^{24}$.

Le témoignage le plus révélateur est celui d'une personnalité laïque de l'époque, l'avocat Gédéon Ouimet, conseiller législatif, surintendant de l'instruction publique, ancien premier ministre québécois. Né en 1823, à Sainte-Rose, dans la seigneurie de l'île Jésus, il entendit très tôt, dès l'âge de huit ou dix ans, sa mère louer $\mathrm{M}^{\mathrm{gr}}$ de Laval. Au cours de ses catéchismes, elle évoquait les sacrifices que coûtèrent au premier évêque de Québec ses longs et pénibles voyages. La brave femme trouvait la vie de $\mathrm{M}^{\mathrm{gr}}$ Plessis - l'évêque de son temps - beaucoup moins difficile que celle de son lointain prédécesseur. Désireuse d'inspirer à son fils, une profonde horreur de l'alcool, elle lui racontait les peines endurées à le combattre par $\mathrm{M}^{\mathrm{gr}}$ de Laval. "Tu vois comment agit un saint," aimait-elle à lui dire. Cette femme, née aux environs de 1780 , ne savait ni lire ni écrire. Toutes ses connaissances elle les tenait d'un enseignement oral reçu de ses parents comme elle l'affirme elle-même: nous avons donc ici les indices d'une tradition orale populaire remontant à la fin du XVIII ${ }^{\circ}$ siècle ${ }^{25}$.

ASQ, Miss 6, verso du $2^{\mathrm{e}}$ feuillet non paginé.

23 ASQ, Summarium 1904, p. 22.

24 ASQ, Summarium 1904, p. 29.

25 ASQ, Mss 439, pp. 133-140. 
Avec la décade de 1840, nous atteignons le sol moins mouvant des sources écrites. Au début de 1842, le Père Tellier, jésuite, avait reçu du duc de Laval-Montmorency une lettre qui lui indiquait la présence, dans son château de Normandie, d'un portrait de $\mathrm{M}^{\mathrm{gr}}$ de Laval. Le P. Tellier communique à Jacques Viger la nouvelle. Celui-ci répond le 13 décembre 1842: "Monsieur le duc de Laval-Montmorenci (sic) sera peut-être flatté d'apprendre que nous avons aussi en Canada tant peint que gravé le portrait de son illustre et saint ancêtre. L'évêché de Québec et le Séminaire de Saint-Sulpice de Montréal l'ont peint sur toile et M. Rouque, prêtre de notre Séminaire, l'a gravé ${ }^{26}$.» Que l'érudition de Viger trébuche, au sujet du nombre des portraits de $M^{\mathrm{gr}}$ de Laval existant au Canada, peu importe, l'intérêt du témoignage n'est pas ici. Le qualificatif « saint » vient sous la plume de Viger tout simplement, sans effort ni arrière-pensée. On pourrait même déceler une nuance d'habitude.

A l'automne de 1845, le Séminaire de Québec donnait l'hospitalité à un jeune prêtre français, l'abbé Etienne-Charles Brasseur de Bourbourg. Il dut inspirer confiance puisque on le chargea immédiatement du cours d'histoire de l'Eglise au Grand Séminaire et qu'on lui demande une biographie de $\mathrm{M}^{\mathrm{gr}}$ de Laval. Le professeur n'eut pas longue carrière: il ne dépassa pas la huitième leçon. (Il ne réussissait pas auprès de ses élèves note Ferland dans ses Observations). L'auteur fut plus' heureux: le 26 décembre 1845, paraissait à Québec l'Esquisse biographique de $M^{\text {or }}$ de Montmorency-Laval, premier évêque de Québec. Le ton polémique de certains passages nous fait soupçonner dans l'Esquisse une réponse prudente et voilée au premier tome de l'Histoire du Canada publié par Garneau cette année-là. L'Almanach ecclésiastique et civil de Québec pour 1846 reproduisit l'Esquisse et le répandit ainsi dans le grand public. Mentionnons pour mémoire au moins la publication anonyme d'une autre $E$ squisse datée de 1845 écrite pourtant semble-t-il en 1847. Nous ne nous attardons pas à celle-ci: le temps nous a manqué pour que nous en puissions parler décemment.

En 1849, on célébra au Séminaire pour la première fois l'anniversaire de $\mathrm{M}^{\mathrm{gr}}$ de Laval. Deux témoins nous ont relaté les faits: l'Abeille et M. Michel-Edouard Méthot, alors sous-diacre. "Lundi dernier dans notre salle de récréation, note le rédacteur de l'Abeille, nous avons célébré avec un plaisir bien marqué et bien sensible, l'anniversaire de naissance de $\mathrm{M}^{\mathrm{gr}}$ de Laval. Outre $\mathrm{M}^{\mathrm{gr}}$ Turgeon et $\mathrm{M}^{\mathrm{gr}}$ Demers (il s'agit ici du premier évêque de Victoria de passage à Québec en route vers Rome), qui nous honoraient de leur présence, il y avait encore plusieurs prêtres... Espérons que chaque année à pareil jour, cette fête se renouvellera pour nous et que nous pourrons nous unir pour payer un tribut d'hommages à notre immortel fondateur ${ }^{27}$." Le 24, mai suivant, Michel Méthot, séminariste, dans une lettre à son oncle, ajoute quelques traits savoureux. Je cite selon une copie à la machine à écrire: l'original a disparu:

«Nous célébrions l'autre jour l'anniversaire de la naissance de $\mathrm{M}^{\mathrm{gr}}$ de Laval. Fête de justice et de reconnaissance qui n'avait pas encore eu

26. ASQ, Fonds Verreau, Boîte 23, liasse 4, n. 3 .

27 L'Abeille, vol. I, n. 32 (3 mai 1849), p. 2. 
lieu au Séminaire de Québec. On avait suspendu le portrait du prélat dans la salle de récréation, on fait de la musique et un petit discours. Le bon vieux évêque (ici indication d'un mot illisible, peut-être prit part) à ces joyeuses démonstration (sic): il paraissait pourtant un peu étonné: il y a si longtemps qu'il était oublié $28 . 》$

L'époque n'était pas prodigue de congés. L'on décide, l'année suivante,

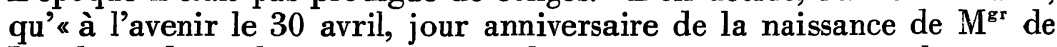
Laval, au dîner des communautés, il y aura une entrée et un dessert et, le soir du même jour, il y aura congé d'études ${ }^{29}$."

C'est à son fondateur que le Séminaire de Québec dédia l'université qu'il fondait en 1852.

1853 et 1874 furent deux années jubilaires qui mirent en plus vive lumière encore la figure de $\mathrm{M}^{\mathrm{gr}}$ de Laval. La première date rappelait le bicentenaire de l'arrivée du prélat au pays; la seconde celui de l'érection du diocèse de Québec. Des fêtes furent célébrées auxquelles un grand concours de peuple prit part. Un détail plein de galanterie fut prévu par le Conseil lors de la manifestation universitaire du 16 juin 1859. "Les dames y seraient admises et occuperaient les galeries (de cette salle) où des sièges rembourés et couverts de toile vernie leur furent réservés ${ }^{30}$.,

L'idée du procès de béatification était dans l'air. A preuve cette lettre de l'abbé Benjamin Paquet à M. Hamel, datée de Rome le 15 mai 1865. "Chaque fois que je suis témoin d'une béatification (la veille il avait assisté à celle da la Bse Marie-des-Anges), je pense toujours à $\mathbf{M}^{\mathrm{gr}}$ de Laval. Nous sommes des ingrats ou des gens de bien peu de foi si nous ne fesons (sic) pas mettre sur les autels le fondateur de l'Eglise du Canada. Je me figure que la cour de Rome serait facile autant qu'elle peut l'être vu que le Nouveau-Monde a bien peu de saints et ensuite vu que $\mathrm{M}^{\mathrm{gr}}$ de Laval est le fondateur d'une église aussi florissante que celle du Canada. Allons à l'œuvre. Commençons par faire faire des miracles à $\mathrm{M}^{\mathrm{gr}}$ de Laval ${ }^{31}$.»

Voici que, le 20 septembre 1877, des menuisiers en réparant le plancher du chœur de la cathédrale, mettent à jour le cercueil de plomb où les restes de $\mathrm{M}^{\mathrm{gr}}$ de Laval reposent depuis 1708. "Cette journée restera célèbre ${ }^{32}$ » remarque, à cette date, l'annaliste du Séminaire. L'émotion n'est pas celle d'une surprise causée par la découverte inattendue d'un trésor, mais celle d'un profond respect envers des ossements pouvant servir un jour de reliques. On le constate aux précautions prises pour en assurer l'authenticité: scellés apposés au coffret où les os sont déposés et à toutes les issues de la chapelle qui' reçoit le coffret, projet d'une translation solennelle. Les autorités du Séminaire se rappelant une clause du testament de $\mathrm{M}^{\mathrm{gr}}$ de Laval qu'il leur avait été impos-

28 ASQ, Correspondance des Recteurs, Boîte 100, n. 52, p. 14.

29 ASQ, Mss 13, T. I, p. 26 verso.

3n ASQ, Mss 13, T. II, p. 5 verso.

31 ASQ, Correspondance des Recteurs; Boîte 104, AW.

32 ASQ, Journal du Séminaire, T. II, p. 663. 
sible de remplir en 1708, demandent de récupérer les restes de leur fondateur. Autorisation leur en est donnée et la translation des restes a lieu très solennellement le 23 mai 1878 non sans que des faveurs signalées ne se produisent sur le parcours du cortège. Les assises du VI concile provincial ont précisément lieu à Québec ces jours-là. Les supérieurs de l'Université Laval et du Séminaire de Québec demandèrent aux pères $d u$ concile $d$ 'appuyer auprès du Saint-Siège leur requête en vue d'obtenir la béatification de $\mathrm{M}^{\mathrm{gr}}$ de Laval. Tous les pères acquiescent et les fidèles sont invités à prier pour le succès de cette cause.

Les procès ordinaire et apostolique tinrent leurs assises: le premier, du 2 mai 1880 au 7 juin 1883; le dernier, du 22 octobre 1898 au 2 avril 1902. Sa Sainteté Léon XIII approuvait l'introduction de la cause en cour de Rome le 24 septembre 1890. Selon la législation canonique du temps, le serviteur de Dieu prenait le titre de Vénérable. La documentation était volumineuse (elle comptait 1285 pages), mais il fut facile au promoteur de la foi d'en déceler la majeure faiblesse: sa mince teneur en documents contemporains (soixante pages de textes). L'avocat de la cause réclama d'autres documents. On lui en envoya fidèlement copiés, mais en nombre insuffisant. Ces lacunes dans l'information firent échouer la cause à la préparatoire de 1918. Une nouvelle documentation partit de Québec.

Sa Sainteté Pie XI avait créé, le 6 mai 1930, au sein de la S. Congrégation des Rites, une section historique destinée aux causes anciennes démunies de témoignages contemporains recueillis juridiquement. $\mathrm{La}$ cause de $\mathrm{M}^{\mathrm{gr}}$ de Laval y passa. On se rendit compte qu'il fallait reprendre $a b$ ovo toute la documentation. Il fallait étudier d'une façon critique chacune des pièces versées au dossier et rechercher dans les divers dépots d'archives, susceptibles d'en contenir, d'autres documents ayant trait au serviteur de Dieu. Il n'est pas étonnant qu'un prêtre du Séminaire vécut sept années à Rome dans ce but. La cause dispose maintenant d'une documentation authentique, fidèle et ample (que l'on songe au 875 pages qu'elle compte maintenant en comparaison des 60 originelles) qui servira de base solide au jugement des consulteurs théologiques. Suivra, nous l'espérons, le décret d'héroïcité des vertus, sanctionné de miracles.

Le long effort humain est parvenu à terme. Restent les desseins secrets de la Providence, où entrent, pour une part, nos prières et nos sacrifices. Comment mieux terminer que par, une pensée de S. S. Pie XII, de regrettée mémoire, exprimée dans un radio-message, lors de la béatification du pape Innocent XI, contemporain de $\mathrm{M}^{\mathrm{gr}}$ de Laval ?

(Dieu) "qui appelle les âmes à une sainteté extraordinaire, se réserve aussi de fixer le jour de leur apothéose selon les desseins d'une infinie sagesse ${ }^{33}$."

Le 11 octobre 1958,

en la fête de la Maternité de Marie.

M. l'abbé Jean-Marie Fortier

Vice-Postulateur de la cause de $M^{g r}$ de Laval.

33 AAS, T. 48 (1956), p. 763. 